\title{
The Epidemiology and Clinical Impact of Surgical Site Infections in the Older Adult
}

\author{
Natasha Bagdasarian • Kenneth E. Schmader • \\ Keith S. Kaye
}

Published online: 1 June 2013

(C) Springer Science+Business Media New York 2013

\begin{abstract}
Surgical site infections (SSI) are the second most frequent healthcare-associated infection (HAI), accounting for approximately $20 \%$ of all HAIs in the United States, and an important cause of morbidity and mortality in older adults. With the aging of the population and the expected increase in surgical procedures in the elderly, strategies for prevention and treatment of SSI, will assume increased importance. In this review we describe risk factors for and outcomes associated with SSI, and recommend treatment and prevention strategies for SSI in older adults.
\end{abstract}

Keywords Elderly $\cdot$ Aged $\cdot$ Surgical Site Infection $\cdot$ Infection

N. Bagdasarian

Division of Infectious Diseases, Department of Medical Education,

Oakwood Healthcare System, Dearborn, MI, USA

N. Bagdasarian $(\varangle)$

Medical Education, Oakwood Hospital and Medical Center,

18101 Oakwood Blvd, Dearborn, MI 48124, USA

e-mail: natasha.bagdasarian@oakwood.org

K. E. Schmader

Division of Geriatrics, Department of Medicine, Duke University

Medical Center, Durham, NC, USA

K. E. Schmader

182 GRECC, Durham VA Medical Center, 508 Fulton Street,

Durham, NC 27705, USA

e-mail: kenneth.schmader@duke.edu

K. S. Kaye

Division of Infectious Diseases, Department of Internal Medicine,

Wayne State University School of Medicine, Detroit, MI, USA

K. S. Kaye

Detroit Medical Center and Wayne State University University

Health Center, 4201 Saint Antoine, Suite 2B,

Box 331, Detroit, MI 48201, USA

e-mail: KKaye@dmc.org

\section{Introduction}

The number of surgeries performed on older adults has been increasing; the proportion of adults aged 65 and over undergoing nonobstetric surgeries was $19 \%$ in 1980 , rising to $43 \%$ in 1998 [1]. To look at one specific example, the annual volume of total knee arthroplasties (TKA) in Medicare recipients increased from 93,230 in 1991 to 243,802 in 2010 [2]. The rates of complex surgeries in older adults may also be on the rise. While rates of spinal surgeries for Medicare recipients from 2002-2007 declined slightly, the rate of complex fusion procedures increased 15 -fold, from 1.3 to almost 20 per 100,000 [3]. These factors contribute to the increased health-care costs for persons aged 65 and older; in the U.S. and other developed countries, health-care costs in older adults are three to five times greater than those for younger individuals $[4,5]$.

Older adults are at increased risk for infection compared to younger adults, due to an increased frequency of comorbid conditions, as well as age-related immune system changes in phagocytosis, cellular migration, and antibody production [6]. When older adults have invasive infections, outcomes tend to be worse compared to younger patients with similar infections. Because older adults represent a vulnerable patient population, prevention of healthcare-associated infections (HAI) in this population is particularly important.

Surgical site infections (SSI) are the second most frequent type of HAI, accounting for approximately $20 \%$ of all HAIs in the United States. National Healthcare Safety Network (NHSN) data for 2006-2008 documented 16,147 SSIs, with an overall SSI rate of $1.9 \%$ [7]. These statistics may be an underestimate given variability in reporting practices [8]. When looking specifically at these outcomes in older adults, Medicare data is sometimes helpful. Of 524,892 eligible Medicare patients who underwent hip 
arthroplasty at 3,296 US hospitals from 2005-2007 (patients with prior major surgery, multiple surgeries during admission and pre-existing hip infection were excluded), chart review of a sample of these patients revealed a variable rate of SSI $(1.2-3.7 \%)[9 \bullet]$.

Given the increased number of surgeries performed on older adults, the need for careful analysis of SSI-associated costs in the older adult, and targeted approaches to reducing these nosocomial infections will assume increased importance. The purpose of this article is to review the definition and surveillance of SSI, describe risk factors and outcomes for SSI in older adults, and recommend treatment and prevention strategies for SSIs in older adults.

\section{Reporting of Surgical Site Infections}

\section{Definitions and Diagnosis}

The National Healthcare Safety Network (NHSN) categorizes SSI as deep incisional SSI, superficial incisional SSI, or organ/space SSI [10]. Superficial incisional SSI occur within 30 days of any operative procedure categorized by NHSN, and involve only the skin and subcutaneous tissue of the incision. Deep incisional SSI occur within 30 days of specific operative procedures (within 90 days if surgery involves an implant), involve fascia and muscle layers, and demonstrate characteristics consistent with infection (ie purulent drainage, evidence of abscess). Organ/space SSI occur within 30 days of specific operative procedures (within 90 days if surgery involves and implant) involve any part of the body that is opened or manipulated during an operative procedure (excluding skin incision, fascia or muscle layers), and again demonstrate characteristics consistent with infection. Clinical signs of infection must be combined with culture and laboratory data to fulfill the diagnosis of SSI. Clinicians should maintain a high index of suspicion for SSI even after hospital discharge, as up to half of all SSI may be diagnosed following hospital discharge [11].

\section{Reporting Practices}

Surveillance for SSI is typically performed by members of a hospital's infection control department. The Center for Medicare and Medicaid Services (CMS) mandates SSI reporting for certain types of procedures via the Centers for Disease Control and Prevention's (CDC) National Healthcare Safety Network (NHSN), as part of their Hospital Inpatient Quality Reporting (IQR) Program. From the beginning of 2006, through the end of 2008, 847 hospitals in 43 states reported a total of 849,659 procedures and 16,147 primary incisional SSIs, among 39 operative procedure categories to the NHSN [7]. The majority of states also have some requirements for SSI reporting to NHSN, which are sometimes more stringent than CMS and CDC requirements [12].

Reporting practices for SSI are not perfect, although the use of Medicare claims for detecting rates of SSI in Medicare recipients, has been shown to be effective $[9 \bullet, 13]$. One study focusing on SSI diagnosed post-discharge, found that $23 \%$ of all related readmissions and $18 \%$ of all emergency room visits due to post-discharge SSI, occurred at institutions other than the index hospital where surgery was performed [14]. In most instances, SSI which are diagnosed at hospitals other than the index institution, are not captured by infection control preventionists at the index hospital.

A study in England found that there was a wide variation in reporting practices of SSI based on the quality of postdischarge surveillance; many sites did not report superficial SSI and $15 \%$ of sites did not use the recommended SSI surveillance definition [15]. The effect of SSI reporting discrepancies may be compounded in older adults, since many older patients are discharged to rehabilitation, or long-term care facilities, where they may receive medical care.

Surveillance is further complicated by subjective factors involved in the diagnosis of SSI. A French study, found that agreement regarding the diagnosis and depth of SSI among healthcare professionals varied across specialties, and amongst individuals in each group, with surprisingly poor consensus among the infection control physicians, and the infection control nurses [16].

\section{Risk Factors for Surgical Site Infection in the Older Adult}

The risk of developing an SSI depends on a multitude of factors, including microbial characteristics (degree of wound contamination, virulence of pathogens), patient characteristics (immune function and comorbidites), and surgical factors (type of procedure, introduction of foreign material) [17]. Risk factors for SSI can be separated into intrinsic patient-associated characteristics, and extrinsic procedurerelated characteristics.

Intrinsic Patient-Associated Risk Factors for Surgical Site Infection

The majority of studies pertaining to SSI risk factors have been performed in the general surgical population, which includes, but is not limited to older adults. Patient characteristics associated with higher risk of SSI deserve close attention, particularly in the elderly, who tend to have a higher frequency of comorbidities than younger persons. In the United States, approximately $80 \%$ of all persons aged $\geq 65$ years have at least one chronic medical condition, and $50 \%$ have at least two chronic conditions $[5,18]$. Diabetes is a traditional risk factor 
for SSI and in 2010 it was estimated to affect 10.9 million, or $26.9 \%$, of adults aged 65 years and older in the U.S. [19].

Patient characteristics traditionally associated with increased risk of SSI in adults of all ages include diabetes mellitus, extremes of age, perioperative hyperglycemia, tobacco use, concomitant infection at another site, obesity, malnutrition, low preoperative serum albumin level, corticosteroid use, prolonged preoperative stay, history of irradiation to the surgical field, impaired immunity, colonization with Staphylococcus aureus and American Society of Anesthesiologists (ASA) physical status classification score of $\geq 3$ [7, 20, 21, 22•].

The data concerning advanced age and the risk of SSI, have been mixed. Several authors have found advanced age to be associated with increased risk for SSI [23-25], while other studies that have found no association between age and risk of surgical site infection [22•,26].

Some interesting associations between age and risk of SSI have also been reported. One study found that advancing age resulted in an increased risk of SSI until age 65 years, after which increasing age predicted a decreased risk of SSI [25]. Although a definitive explanation for this finding was not provided, the authors hypothesized that it may have been secondary to a "hardy survivor" effect, wherein older patients had physiologic advantages compared to younger patients, which decreased their risk of SSI; or to selection bias, where invasive surgeries were less frequently offered to "high-risk" older adults with severe underlying comborbid conditions.

There have been a few studies focusing specifically on SSI in older adults. A review of 569 cases of SSI in older patients ( $\geq 65$ years) confirmed that COPD, obesity, and the presence of a contaminated or dirty wound were independent predictors of SSI [27]. Pre-operative admission to the hospital from a healthcare facility, has been reported to be a risk factor for SSI in older patients following orthopedic surgery [28]. Notably, in this study of 169 adults $\geq 75$ years of age, comorbid conditions including morbid obesity, duration of surgery, and perioperative hyperglycemia were not found to be significant risk factors for SSI [28]. Factors reported to be associated with a decreased risk for SSI in older adults include ownership of private health insurance, and having surgery performed on the day of hospital admission [27, 29]. The authors postulated that private insurance may serve as a marker for patients with increased access to healthcare services, or community support systems.

Extrinsic Procedural Risk Factors for Surgical Site Infection

Increased risk of SSI has classically been associated with several procedural characteristics in the general surgical population, including lack of preoperative antiseptic bathing, shaving of surgical site the night before procedure, use of a razor for hair removal, improper preoperative skin preparation, improper antimicrobial prophylaxis, failure to re-dose antibiotics during prolonged procedures, inadequate operating room (OR) ventilation, increased OR traffic, break in aseptic technique, perioperative hypothermia or hypoxia, surgery classified as contaminated or dirty/infected, or a procedure lasting longer than the duration cut point time assigned to that type of operation $[7,20,30]$. Surgical skill, and the facility in which a surgical procedure is performed, can lead to significant variability in the subsequent risk of SSI [9].

The NHSN has devised an index to predict the risk of SSI, which takes into account some of these patient and procedural factors. The risk index is scored from 0 to 3 , and points are assigned based on the presence of the following factors: American Society of Anesthesiologists' physical status classification score of $\geq 3$, surgery classified as contaminated or dirty/infected, or a procedure lasting longer than the duration cut point time assigned to that type of operation [7]. Interestingly, the NHSN risk index does not take advanced age into account.

Risk Factors for Surgical Site Infections due to Staphylococcus aureus in the Older Adult

One of the most common pathogens implicated in SSI is Staphylococcus aureus, and in several reports, Methicillinresistant $S$. aureus (MRSA) has been the single most common pathogen causing invasive SSI $[31 \cdot, 32]$. Reports suggest that higher rates of invasive $S$. aureus infections occur after cardiothoracic procedures, versus some other types of surgical procedures [32].

MRSA has emerged as a leading cause of SSI, surpassing methicillin-susceptible $S$. aureus (MSSA) in frequency in many settings [32, 33]. Some risk factors which have been associated with SSI due to MRSA in patients over the age of 65 include poor functional status, increased Charlson comorbidity score and wound class $>1[31 \bullet]$. The authors postulated that subjects who required assistance with bathing or dressing likely had more frequent and intensive contact with the healthcare system and healthcare providers and were at increased risk of preoperative MRSA colonization.

\section{Treatment of Surgical Site Infections in the Older Adult}

Superficial incisional SSIs are often managed in the outpatient setting; however deep incisional and organ/space infections typically require readmission to the hospital for management [34]. There are a few basic concepts to treating SSI in the elderly, which will be discussed below.

For deep/incisional and organ/space infections, effective drainage of abscesses and/or debridement of devitalized tissue is vitally important. Antimicrobial therapy is an important adjuvant to debridement. Detailed recommendations pertaining to antimicrobial therapy for different types of SSI are reviewed in detail elsewhere [35]. For superficial incisional SSI, duration 
of antimicrobials is usually 7-10 days. For deep incisional and organ/space SSI, duration of therapy is calculated beginning with the day of definitive abscess drainage or debridement. Typical antimicrobial treatment durations for deep incisional SSI are 2 weeks following debridement; and for organ/space infection, 2-6 weeks following debridement. However treatment course will vary depending on the depth, location and extent of infection, as well as presence of prosthetic material.

Infections of prosthetic joints (PJI) present a particular challenge for both clinicians and patients. Definitive cure of PJI typically requires removal of the prosthesis, preservation of the joint space, and systemic antimicrobial therapy for 4-6 weeks [36]. After therapy has been complete and it is apparent that the patient has responded to therapy, a new prosthesis is usually placed. This prolonged treatment course and multiple surgical procedures can have a huge toll on older adults, and is associated with significant adverse impact on a patient's clinical status; functional status can be significantly compromised. If certain clinical and microbologic criteria are met, another treatment approach involving retention of the prosthesis with debridement and prolonged antimicrobial therapy can be attempted [36]. PJI are serious and challenging infections to treat, particularly in older adults, and it is recommended to involve an infectious diseases specialist to help comanage these cases.

There are many inherent challenges with the management of SSI in older adults. One of the most important concepts for clinicians managing older adults who have SSI (particularly SSIs categorized as deep incisional or organ/space) is to balance risk associated with prolonged antimicrobial therapy and repeated surgeries with the preservation of functional status. For example, treatment of organ/space SSI or PJI may require prolonged courses of intravenous antimicrobial therapy, which can raise issues related to the safety and tolerability of antimicrobial agents. Prolonged courses of antibiotics in older, post-surgical patients result in increased the risk of Clostridium difficile infections (CDI), which is in itself a significant cause of morbidity and mortality in the elderly. Thus, careful consideration and review of treatment options, often in conjunction with surgical, geriatric and infectious diseases specialists, should be undertaken in these situations.

The aging process can result in changes in metabolism and excretion of medications. Renal blood flow decreases with age, resulting in a $30 \%-50 \%$ decrease in creatinine clearance between the ages of 20-90 years of age. Liver mass and hepatic blood flow also decrease as individuals age, leading to changes in the metabolism and excretion of medications, including antimicrobial agents [37]. Clinicians should be mindful of these factors when prescribing antibiotics to older adults.

As the incidence of MRSA infections has risen, vancomycin has been used more frequently for treatment and at higher doses. Clinicians should be aware that these higher doses of vancomycin (serum trough levels of $15-20 \mathrm{mg} / \mathrm{dl}$ ) and prolonged durations of therapy may be associated with an increased risk of nephrotoxicity [38].

Polypharmacy can be an issue in older surgical patient, especially when new medications prescribed during the postoperative period are added to the patient's chronic medications. In one report, almost one quarter of surgical patients over the age of 65 years received potentially inappropriate medications, as determined by an expert panel, which could be associated with a high risk of adverse events in older adults [39].

\section{Outcomes of Older Adults with Surgical Site Infections}

\section{Duration and Costs of Hospitalization}

SSI is associated with an increase in postoperative duration of hospitalization and healthcare costs. A case of SSI is associated with approximately 7-10 additional post-operative hospital days [40-42]. Length of stay may be even higher among older patients, with one report showing a mean attributable duration of hospitalization after surgery exceeding 15 days, and mean attributable hospital charges of $\$ 43,970$ in patients aged 65 years and older [43••]. Older adults with SSI have been reported to have an almost threefold longer duration of postoperative hospitalization and an almost twofold increase in higher hospital charges compared to older surgical patients without SSI [43••].

Attributable costs can vary depending on the surgical procedure, pathogen, as well as extent and severity of infection [44-46], however SSI are annually believed to account for up to $\$ 7$ billion in health care expenditures [47].

\section{Mortality}

Operative patients with SSI may have twice the mortality rate, compared to noninfected patients [40]. In older adults this effect is amplified, with SSI resulting in an almost fourfold increase in mortality [43••].

SSI is independent predictor of one-year mortality in older adults who underwent orthopedic surgery [28]. An outcomes study in patients aged 65 years and older with SSI following orthopedic surgery, identified the following predictors of mortality: age 75 years or older, admission from a health-care facility, Charlson score $\geq 3$, congestive heart failure, cerebrovascular accident, COPD, an ASA score of $\geq 3$, and inability to independently perform activities of daily living [28]. Hospital admission on the day of the surgery was associated with a decreased risk of one-year mortality [28].

Functional Status

Functional status has been identified as a risk factor for the development of SSI in older adults, but as an outcome, this 
issue has not been well studied. While SSI poses challenges in the recovery of older operative patients, those who survive the post-operative period may have an improved quality of life. As the frequency of surgeries in older adults increases, determining the impact of SSI on functional status and identifying modifiable risk factors to prevent declines in functional status following SSI are important areas of future research.

\section{Outcomes Associated with SSI Due to $S$. aureus}

S. aureus infections are associated with significantly increased morbidity and mortality, and older adults with infections due to $S$. aureus generally experience worse clinical outcomes than do younger ones. In one report of patients over the age of 70 years, SSI due to $S$. aureus was associated with a fivefold increase in mortality, a two-fold increase in duration of postoperative hospital length of stay, and excess attributable hospital costs of over $\$ 40,000$ per SSI, compared to uninfected older surgical patients [48].

MRSA in particular often results in worse outcomes and increased healthcare costs compared to MSSA [32, 33]. Anderson et al. reported that compared to uninfected controls, patients in the general surgical population with SSI due to MRSA, had a sevenfold increased risk of death, a 35-fold increased risk of hospital readmission, more than 3 weeks of additional hospitalization, and over $\$ 60,000$ of additional charges [33].

\section{Prevention of Surgical Site Infections in the Older Adult}

While there are no guidelines for the prevention of SSI specifically for older adults, basic tenants of prevention in the general population are often applicable. Meticulous approaches to SSI prevention described below are necessary to optimize the outcomes of the older operative patient.

\section{Antibiotic Prophylaxis}

A detailed discussion of antimicrobial prophylaxis is beyond the scope of this article and has been discussed in detail elsewhere [49]. Preoperative antibiotics should be selected based on the type of surgical procedure, the most common pathogens causing SSI for that type of specific procedure, and published recommendations [30, 49].

Antibiotic prophylaxis should be administered within one hour before incision to maximize tissue concentration at the site of surgery; vancomycin and fluoroquinolones can be administered within 2 hours of incision [30, 49]. Guidelines suggest stopping prophylactic antimicrobials within 24 hours following surgery, as there is no evidence that continued therapy following closure is beneficial, and prolonged antibiotics contribute to increased adverse events including $C$. difficile infection and antimicrobial resistance [50, 51].

Antibiotic dosing is an important factor when considering antimicrobial prophylaxis. Antibiotic dosing should take into account patient weight, particularly in obese patients, and antibiotics should be redosed during lengthy procedures [17, 49]. A combination of parenteral antibiotics, preoperative mechanical bowel preparation, and oral antibiotics should be used to reduce the risk of SSI following elective colorectal procedures $[17,52,53]$.

\section{Other Recommendations}

Several other recommendations for prevention of SSI are detailed as part of the Surgical Care Improvement Project (SCIP), which is a national multi-agency collaborative effort to reduce surgical complications (REF). The Center for Medicare and Medicaid Services (CMS) requires hospitals to submit data on SCIP measures as a part of the Hospital Inpatient Quality Reporting System (IQR), including antimicrobial prophylaxis.

Recommendations for the prevention of SSI include removal of hair only if the presence of hair will interfere with the operation; and if removal is necessary, that hair is removed by means other than a razor (i.e., via clipping or depilatory creams) [17]. Control of blood glucose during the immediate postoperative period for cardiac surgery patients is recommended to reduce the risk of SSI $[17,30,54]$. Maintaining normothermia (temperature $\geq 35.5{ }^{\circ} \mathrm{C}$ ) is recommended for colorectal procedures, well as other types of operative procedures [17, 55]. These last two concepts may be of particular importance when caring for older patients given the high rate of diabetes in older adults [19], as well as age-associated differences in thermal homeostasis [56].

\section{Unanswered Questions}

There are many unanswered questions in the area of SSI prevention, and while these issues do not pertain only to older adults, these concepts are important in the management of older patients, who tend to have higher rates of comorbid conditions, and reside in long-term care settings, where there might be an increased risk for colonization with, and infection due to, MRSA.

\section{Screening and Decolonization of S. aureus and MRSA}

There are data supporting the use of nasal mupirocin to prevent SSI due to $S$. aureus in persons colonized with intranasally with S. aureus in the preoperative period [57]. Clinical practice guidelines from America Society of Health-System Pharmacists (ASHP) recommend that mupirocin should be given intranasally to all patients with documented $S$. aureus colonization for 
orthopedic and cardiac procedures [58]. A randomized, doubleblind, placebo-controlled, trial that evaluated treatment with mupirocin nasal ointment and chlorhexidine soap in $S$. aureus nasal carriers, was associated with a reduction in the risk for post-operative infection due to $S$. aureus and deep SSI due to $S$. aureus [59].

A study evaluating a prevention protocol for SSI due to MRSA involved a 5-day course of intranasal mupirocin and use of $2 \%$ chlorhexidine gluconate cloths, in neurosurgical, orthopedic, vascular and cardiac surgery patients; this intervention was associated with a decreased risk for SSI due to MRSA [60]. However, for certain types of procedures, decolonization of $S$. aureus has not had a significant impact on SSI. Huttner et al. reported that MRSA was an infrequent cause of SSI after colorectal resections, even among MRSA carriers, and that preoperative screening for MRSA carriage and decolonization of MRSA carriers, would not be effective [61].

\section{Prophylaxis with Vancomycin}

Recent guidelines recommend limiting vancomycin use for antimicrobial prophylaxis to only specific circumstances, such as during outbreaks of MRSA, when endemic rates of SSI due to MRSA are "increased", and for patients who are at increased risk of SSI due to MRSA [17]. However there are no clear definitions for what constitutes an "increased" endemic rate of SSI due to MRSA, and studies on the efficacy of vancomycin prophylaxis were published prior to the emergence of community-acquired MRSA [17]. Vancomycin, if administered alone as a prophylactic agent, has been reported to increase the risk for SSI due to methicillin-susceptible pathogens, such as MSSA [62].

\section{Conclusion}

Although surgical procedures can improve the quality and duration of life of older adults, SSI is an important cause of morbidity and mortality, as well as increased health-care costs. The need for careful analysis of SSI-associated costs in the older adult and targeted approaches to reducing these nosocomial infections will assume increased importance as the population continues to age. Treatment of deep incisional and organ/space SSI often requires additional procedures and prolonged courses of antimicrobial therapy. When caring for an older adult with a deep-seated SSI, the risks and benefits of different therapeutic approaches should be carefully considered, ideally with input from surgical, infectious diseases and geriatric specialists.

\section{Compliance with Ethics Guidelines}

Conflict of Interest Natasha Bagdasarian declares that she has no conflict of interest.
Kenneth E. Schmader declares that he has no conflict of interest. Keith S. Kaye declares that he has no conflict of interest.

Human and Animal Rights and Informed Consent This article does not contain any studies with human or animal subjects performed by any of the authors.

\section{References}

Papers of particular interest, published recently, have been highlighted as:

- Of importance

•- Of major importance

1. Popovic JR, Kozak LJ. National hospital discharge survey: annual summary, 1998. Vital Health Stat. 2000;13(148):1-194.

2. Cram P, Lu X, Kates SL, et al. Total knee arthroplasty volume, utilization, and outcomes among Medicare beneficiaries, 19912010. JAMA. 2012;308(12):1227-36.

3. Deyo RA, Mirza SK, Martin BI, et al. Trends, major medical complications, and charges associated with surgery for lumbar spinal stenosis in older adults. JAMA. 2010;303(13):1259-65.

4. Anderson GF, Hussey PS. Population aging: a comparison among industrialized countries. Health Aff (Millwood). 2000;19(3):191203.

5. Trends in aging-United States and worldwide. MMWR Morb Mortal Wkly Rep. 2003 Feb 14;52(6):101-4, 6.

6. Agarwal S, Busse PJ. Innate and adaptive immunosenescence. Ann Allergy Asthma Immunol. 2010;104(3):183-90. quiz 90-2, 210.

7. $\mathrm{Mu} \mathrm{Y,} \mathrm{Edwards} \mathrm{JR,} \mathrm{Horan} \mathrm{TC,} \mathrm{et} \mathrm{al.} \mathrm{Improving} \mathrm{risk-adjusted}$ measures of surgical site infection for the national healthcare safety network. Infect Control Hosp Epidemiol. 2011;32(10):970-86.

8. Klevens RM, Edwards JR, Richards Jr CL, et al. Estimating health care-associated infections and deaths in U.S. hospitals, 2002. Public Health Rep. 2007;122(2):160-6.

9. - Calderwood MS, Kleinman K, Bratzler DW, et al. Use of Medicare claims to identify US hospitals with a high rate of surgical site infection after hip arthroplasty. Infect Control Hosp Epidemiol. 2013;34(1):31-9. Retrospective cohort study in which Medicare claims were successfully used to identify US hospitals with high rates of surgical site infection (SSI) after hip arthroplasty.

10. Mangram AJ, Horan TC, Pearson ML, et al. Guideline for prevention of surgical site infection, 1999. Hospital Infection Control Practices Advisory Committee. Infect Control Hosp Epidemiol. 1999;20(4):250-78. quiz 79-80.

11. Daneman N, Lu H, Redelmeier DA. Discharge after discharge: predicting surgical site infections after patients leave hospital. J Hosp Infect. 2010;75(3):188-94.

12. [cited 2013]; Available from: http://www.hospitalinfection.org/ legislation.

13. Haley VB, Van Antwerpen C, Tserenpuntsag B, et al. Use of administrative data in efficient auditing of hospital-acquired surgical site infections, New York State 2009-2010. Infect Control Hosp Epidemiol. 2012;33(6):565-71.

14. Perencevich EN, Sands KE, Cosgrove SE, et al. Health and economic impact of surgical site infections diagnosed after hospital discharge. Emerg Infect Dis. 2003;9(2):196-203.

15. Tanner J, Padley W, Kiernan M, et al. A benchmark too far: findings from a national survey of surgical site infection surveillance. J Hosp Infect. 2013;83(2):87-91. 
16. Lepelletier D, Ravaud P, Baron G, et al. Agreement among health care professionals in diagnosing case Vignette-based surgical site infections. PLoS One. 2012;7(4):e35131.

17. Anderson DJ, Kaye KS, Classen D, et al. Strategies to prevent surgical site infections in acute care hospitals. Infect Control Hosp Epidemiol. 2008;29 Suppl 1:S51-61.

18. Chronic disease notes and reports: special focus. Healthy Aging 1999;12:3. http://www.cdc.gov/aging/pdf/CDNR.June.2007.pdf

19. 2013; Available from: http://www.diabetes.niddk.nih.gov/dm/pubs/ statistics/.

20. Talbot TR, Schaffner W. Relationship between age and the risk of surgical site infection: a contemporary reexamination of a classic risk factor. J Infect Dis. 2005;191(7):1032-5.

21. Richards JE, Kauffmann RM, Zuckerman SL, et al. Relationship of hyperglycemia and surgical-site infection in orthopaedic surgery. J Bone Joint Surg Am. 2012;94(13):1181-6.

22. - Namba RS, Inacio MC, Paxton EW. Risk factors associated with surgical site infection in 30,491 primary total hip replacements. J Bone Joint Surg Br. 2012;94(10):1330-8. A retrospective review of a cohort of primary total hip replacement (THR) performed between 2001 and 2009, to identify patient and surgical factors associated with deep surgical site infection (SSI) following THR.

23. de Boer AS, Mintjes-de Groot AJ, Severijnen AJ, et al. Risk assessment for surgical-site infections in orthopedic patients. Infect Control Hosp Epidemiol. 1999;20(6):402-7.

24. Scott JD, Forrest A, Feuerstein S, et al. Factors associated with postoperative infection. Infect Control Hosp Epidemiol. 2001;22(6):347-51.

25. Kaye KS, Schmit K, Pieper C, et al. The effect of increasing age on the risk of surgical site infection. J Infect Dis. 2005;191(7):1056-62.

26. Malone DL, Genuit T, Tracy JK, et al. Surgical site infections: reanalysis of risk factors. J Surg Res. 2002;103(1):89-95.

27. Kaye KS, Sloane R, Sexton DJ, et al. Risk factors for surgical site infections in older people. J Am Geriatr Soc. 2006;54(3):391-6.

28. Lee J, Singletary R, Schmader K, et al. Surgical site infection in the elderly following orthopaedic surgery. Risk factors and outcomes. J Bone Joint Surg Am. 2006;88(8):1705-12.

29. Kaye KS, Schmader KE, Sawyer R. Surgical site infection in the elderly population. Clin Infect Dis. 2004;39(12):1835-41.

30. Bratzler DW, Hunt DR. The surgical infection prevention and surgical care improvement projects: national initiatives to improve outcomes for patients having surgery. Clin Infect Dis. 2006;43(3):322-30.

31. Chen TY, Anderson DJ, Chopra T, et al. Poor functional status is an independent predictor of surgical site infections due to methicillin-resistant Staphylococcus aureus in older adults. J Am Geriatr Soc. 2010;58(3):527-32. Risk factors for MRSA SSI were identified by comparing cases with uninfected surgical patients and patients with SSI due to MSSA.

32. Anderson DJ, Arduino JM, Reed SD, et al. Variation in the type and frequency of postoperative invasive Staphylococcus aureus infections according to type of surgical procedure. Infect Control Hosp Epidemiol. 2010;31(7):701-9.

33. Anderson DJ, Kaye KS, Chen LF, et al. Clinical and financial outcomes due to methicillin resistant Staphylococcus aureus surgical site infection: a multi-center matched outcomes study. PLoS One. 2009;4(12):e8305.

34. Ming DY, Chen LF, Miller BA, et al. The impact of depth of infection and postdischarge surveillance on rate of surgical-site infections in a network of community hospitals. Infect Control Hosp Epidemiol. 2012;33(3):276-82.

35. May AK. Skin and soft tissue infections: the new surgical infection society guidelines. Surg Infect (Larchmt). 2011;12(3):179-84.

36. Osmon DR, Berbari EF, Berendt AR, et al. Diagnosis and management of prosthetic joint infection: clinical practice guidelines by the infectious diseases society of america. Clin Infect Dis. 2013;56(1):e1-e25.
37. White PF, White LM, Monk T, et al. Perioperative care for the older outpatient undergoing ambulatory surgery. Anesth Analg. 2012;114(6):1190-215.

38. Pritchard L, Baker C, Leggett J, et al. Increasing vancomycin serum trough concentrations and incidence of nephrotoxicity. Am J Med. 2010;123(12):1143-9.

39. Finlayson E, Maselli J, Steinman MA, et al. Inappropriate medication use in older adults undergoing surgery: a national study. J Am Geriatr Soc. 2011;59(11):2139-44.

40. Kirkland KB, Briggs JP, Trivette SL, et al. The impact of surgicalsite infections in the 1990s: attributable mortality, excess length of hospitalization, and extra costs. Infect Control Hosp Epidemiol. 1999;20(11):725-30.

41. Cruse PJ, Foord R. The epidemiology of wound infection. A 10year prospective study of 62,939 wounds. Surg Clin North Am. 1980;60(1):27-40.

42. Cruse P. Wound infection surveillance. Rev Infect Dis. 1981;3(4):734 7.

43. • Kaye KS, Anderson DJ, Sloane R, et al. The effect of surgical site infection on older operative patients. J Am Geriatr Soc. 2009;57(1):46-54. Retrospective matched-outcomes study comparing older post-operative patients with deep incisional or organ/space SSI to operative patients without SSI. This study found increased mortality, length of stay and healthcare costs in elderly patients with SSI.

44. Coello R, Glenister H, Fereres J, et al. The cost of infection in surgical patients: a case-control study. J Hosp Infect. 1993;25(4):239 50.

45. Hollenbeak CS, Murphy DM, Koenig S, et al. The clinical and economic impact of deep chest surgical site infections following coronary artery bypass graft surgery. Chest. 2000;118(2):397402.

46. Whitehouse JD, Friedman ND, Kirkland KB, et al. The impact of surgical-site infections following orthopedic surgery at a community hospital and a university hospital: adverse quality of life, excess length of stay, and extra cost. Infect Control Hosp Epidemiol. 2002;23 (4):183-9.

47. Skoufalos A, Clarke JL, Napp M, et al. Improving awareness of best practices to reduce surgical site infection: a multistakeholder approach. Am J Med Qual. 2012;27(4):297-304.

48. McGarry SA, Engemann JJ, Schmader K, et al. Surgical-site infection due to Staphylococcus aureus among elderly patients: mortality, duration of hospitalization, and cost. Infect Control Hosp Epidemiol. 2004;25(6):461-7.

49. Bratzler DW, Dellinger EP, Olsen KM, et al. Clinical practice guidelines for antimicrobial prophylaxis in surgery. Surg Infect (Larchmt). 2013;14(1):73-156.

50. Harbarth S, Samore MH, Lichtenberg D, et al. Prolonged antibiotic prophylaxis after cardiovascular surgery and its effect on surgical site infections and antimicrobial resistance. Circulation. 2000;101 (25):2916-21.

51. Coakley BA, Sussman ES, Wolfson TS, et al. Postoperative antibiotics correlate with worse outcomes after appendectomy for nonperforated appendicitis. J Am Coll Surg. 2011;213(6):778-83.

52. Fry DE. Colon preparation and surgical site infection. Am J Surg. 2011;202(2):225-32.

53. Nelson RL, Glenny AM, Song F. Antimicrobial prophylaxis for colorectal surgery. Cochrane Database Syst Rev. 2009;1, CD001181.

54. Lazar HL, McDonnell M, Chipkin SR, et al. The Society of Thoracic Surgeons practice guideline series: blood glucose management during adult cardiac surgery. Ann Thorac Surg. 2009; 87(2):663-9.

55. Kurz A, Sessler DI, Lenhardt R. Perioperative normothermia to reduce the incidence of surgical-wound infection and shorten hospitalization. Study of Wound Infection and Temperature Group. N Engl J Med. 1996;334(19):1209-15. 
56. Blatteis CM. Age-dependent changes in temperature regulation - a mini review. Gerontology. 2012;58(4):289-95.

57. van Rijen M, Bonten M, Wenzel R, et al. Mupirocin ointment for preventing Staphylococcus aureus infections in nasal carriers. Cochrane Database Syst Rev. 2008;4, CD006216.

58. Bratzler DW, Dellinger EP, Olsen KM, et al. Clinical practice guidelines for antimicrobial prophylaxis in surgery. Am J Health Syst Pharm. 2013;70(3):195-283.

59. Bode LG, Kluytmans JA, Wertheim HF, et al. Preventing surgical-site infections in nasal carriers of Staphylococcus aureus. N Engl J Med. 2010;362(1):9-17.
60. Thompson P, Houston S. Decreasing methicillin-resistant Staphylococcus aureus surgical site infections with chlorhexidine and mupirocin. Am J Infect Control. 2013. doi:10.1016/j.ajic.2012.09.003

61. Huttner B, Robicsek AA, Gervaz P, et al. Epidemiology of methicillin-resistant Staphylococcus aureus carriage and MRSA surgical site infections in patients undergoing colorectal surgery: a cohort study in two centers. Surg Infect (Larchmt). 2012;13(6):401-5.

62. Bull AL, Worth LJ, Richards MJ. Impact of vancomycin surgical antibiotic prophylaxis on the development of methicillin-sensitive staphylococcus aureus surgical site infections: report from Australian Surveillance Data (VICNISS). Ann Surg. 2012;256(6):1089-92. 